\title{
石墨电极首次阴极极化过程的两电极和三电极电化学 阻抗谱研究
}

庄全超 ${ }^{(\mathbb{*} *}$, 田雷雷 ${ }^{(1)}$, 魏国祯 ${ }^{(2}$, 董全峰 ${ }^{(2}$, 孙世刚 ${ }^{(*}$

(1) 中国矿业大学材料科学与工程学院, 徐州 221116;

(2) 固体表面物理化学国家重点实验室, 厦门大学化学化工学院化学系, 厦门 361005

* 联系人, E-mail: zhuangquanchao@126.com, sgsun@xmu.edu.cn

国家重点基础研究发展计划(编号: 2009CB220102)资助项目

摘要 运用电化学阻抗谱(EIS)并结合扫描电子显微镜(SEM)研究了石墨电极在两电极扣式 电池和三电极玻璃电解池中的首次阴极极化过程. 研究指出, 两电极扣式电池体系中石墨 电极首次阴极极化过程的 EIS 谱特征及其变化不能解释固体电解质相界面膜(SEI 膜)的形成 过程, 但是可以用三电极玻璃电解池中获得的石墨电极首次阴极极化的 EIS 谱特征及其变 化加以说明. 结果表明, 在石墨负极首次阴极极化过程中 SEI 膜主要在 $1.0 \sim 0.6 \mathrm{~V}$ 电位区间

关键词 锂离子电池 石墨电极 电化学阻抗谱 SEI 膜 形成.

电化学阻抗谱(electrochemical impedance spectroscopy, EIS) 是研究电极/电解质界面过程的最有力 工具之一. 在过去的十多年里, EIS被广泛地应用于 研究锂离子在碳材料和过渡金属氧化物中的嵌入过 程 ${ }^{[1 \sim 5]}$. 根据Aurbach研究小组 ${ }^{[6]}$ 的观点, 锂离子嵌入 嵌合物电极的过程主要包括以下几个步骤: 锂离子 在电解液中的扩散、锂离子通过SEI膜(固体电解质相 界面膜)的迁移、电荷传递和锂离子在石墨电极中的 固态扩散. EIS能够根据上述过程中每一步骤弛豫时 间常数的不同, 而在较宽频率范围内表征上述过程 中的每一步骤. 在EIS实际应用中面临的一个主要问 题是其不确定性, 主要表现为很多不同的物理过程 或一个复杂过程的不同步骤呈现为相似的阻抗谱特 征. 经典的电化学体系通常包括电荷传递、扩散和还 原产物的吸附等过程, 如果所有吸附位置具有相同 的位能, 也就是说电极的表面是能量均匀的, 那么对 上述过程的区分是非常容易的. 然而与上述经典电 化学体系相比, 在嵌锂电极中发生的过程要复杂
得多, 这主要是因为嵌锂电极通常都是由黏合剂、活 性材料、导电剂和集流体组成的复合电极, 嵌锂电极 的组成和活性材料颗粒的大小以及电极的厚度和制 备工艺等诸多因素均会对阻抗谱的特征产生重要的 影响 ${ }^{[7,8]}$. 因此通过详细的对比实验确定EIS谱中每一 时间常数对应的物理过程是非常重要的.

在本文中, 我们运用 EIS 并结合扫描电子显微镜 (SEM)研究了石墨电极在两电极扣式电池和三电极玻 璃电解池中的首次阴极极化过程, 重点探讨电化学 阻抗谱高频区域半圆的归属问题.

\section{1 实验}

实验在自制三电极玻璃电解池和两电极扣式电 池中完成, 金属锂片作为参比和辅助电极. 石墨电极 由 $90 \%$ 的石墨碳纤维 (mesophase-pitch-based carbon fibers, MCF. Petoca, Japan)和 10\%的 PVDF-HFP (Kynar FLEX 2801, Elf-atochem, USA)黏合剂组成. 电解液为 $1 \mathrm{~mol} \cdot \mathrm{L}^{-1} \mathrm{LiPF}_{6}-\mathrm{EC}($ 碳酸乙 烯酯 $): \operatorname{DEC}($ 碳酸二乙 
酯): DMC(碳酸二甲酯)(1:1:1, 体积比)(张家港国泰 华荣化工新材料公司).

所有电化学测试均在电化学工作站(CHI660B, 上海辰华仪器厂)上完成. 电化学阻抗测试频率范围 为 $10^{5} \sim 10^{-2} \mathrm{~Hz}$, 施加的交流信号振幅为 $5 \mathrm{mV}$. 在进 行阻抗测试前, 电极在极化电位平衡 $1 \mathrm{~h}$. 电极形貌 用英国 Oxford Instrument 公司生产的 LEO 1530 型场 发射电子显微镜进行观察.

\section{2 结果与讨论}

\subsection{SEM 研究结果}

图 1 为石墨电极经历电化学扫描循环前不同放 大倍数的 SEM 图，显示本文采用的石墨材料为棒状 的石墨化碳纤维及其碎块, 具有明显的层状结构. 在 经历电化学扫描循环前, 其表面不存在 SEI 膜. 图 2 为石墨电极经历电化学扫描循环后不同放大倍数的 SEM 图, 其表面形貌发生了很大的变化, 明显为一 层表面层(即 SEI 膜)所覆盖.

\section{2 石墨电极首次阴极极化过程的 EIS 研究}

图 3 显示石墨电极在 $1 \mathrm{~mol} \cdot \mathrm{L}^{-1} \mathrm{LiPF} 6-\mathrm{EC}$ ：DEC：
DMC电解液中首次阴极极化过程中 Nyquist图随电极 电位(3.0 1.0 V)的变化. 从图 3(a)可以看出, 在两电 极扣式电池中石墨电极在其开路电位 $(3.0 \mathrm{~V})$ 的 Nyquist图由两部分组成, 即高频区域的一个半圆 (high frquency arc, HFA)和低频区域的一段圆弧. 这 与Holzapfel等人 ${ }^{[9]}$ 在三电极扣式电池中的研究结果 相一致，表明在扣式电池体系中，是否使用单独的金 属锂片作为参比电极对EIS的谱特征影响较小. 在电 极极化电位降低的过程中, 当电位大于 $1.5 \mathrm{~V}$ 时, HFA 不断增大; 而在 1.5 1.1 V之间基本保持不变. 已有 研究表明 ${ }^{[6,10 ~ 12]}$, HFA是由石墨电极表面的SEI膜所致. 如果认为极化电位降低至 $1.1 \mathrm{~V}$ 以前, EIS的Nyquist图 中的HFA主要与SEI膜相关, 那么则存在以下两点无 法解释: (1) 根据SEM的研究结果, 石墨电极在经历 电化学扫描循环前, 其表面不存在SEI 膜, 因此石墨 电极首次阴极极化过程中在开路电位时, EIS 的 Nyquist图中应该不存在HFA; (2) 根据电化学循环伏 安法(CV)的研究结果可知 ${ }^{[13]}$, SEI膜主要在 1.0 0.5 V 之间形成, 然而 $3.0 \sim 1.5 \mathrm{~V}$ 之间, H F A 却
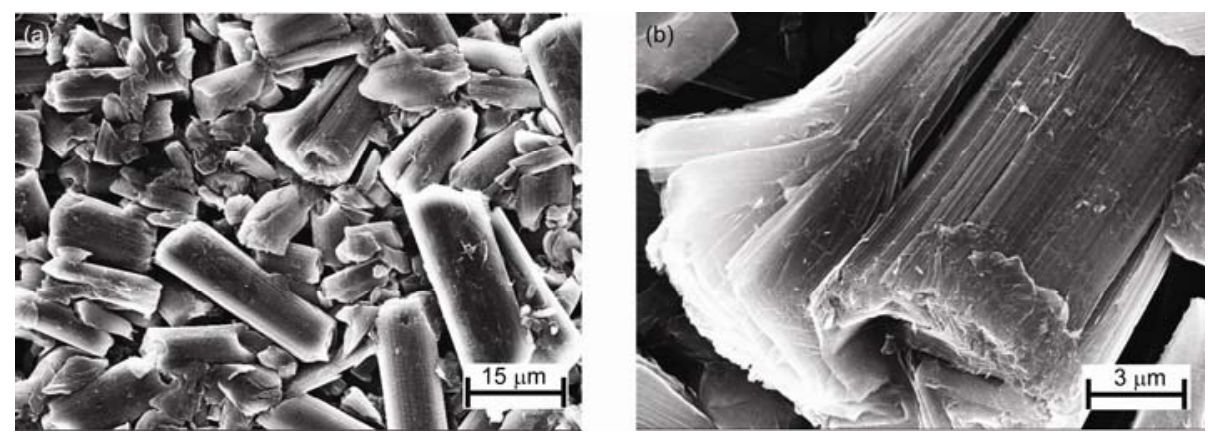

图 1 石墨电极经历电化学扫描循环前不同放大倍数的 SEM 图 (a) 1000 倍; (b) 5000 倍
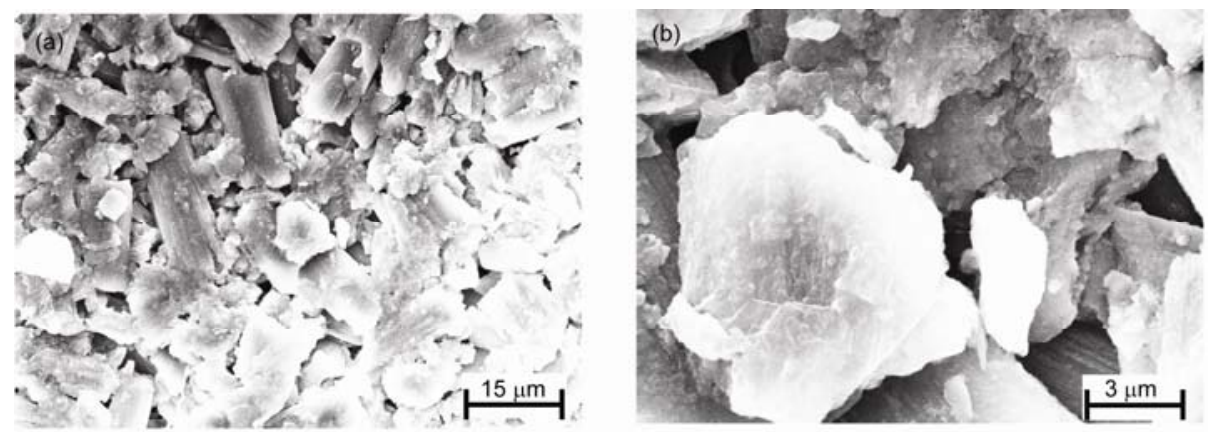

图 2 石墨电极在 $1 \mathrm{~mol} \cdot \mathrm{L}^{-1} \mathrm{LiPF}_{6}-\mathrm{EC}$ : DEC: DMC 电解液中经历电化学扫描循环后不同放大倍数的 SEM 图 (a) 1000 倍; (b) 5000 倍 

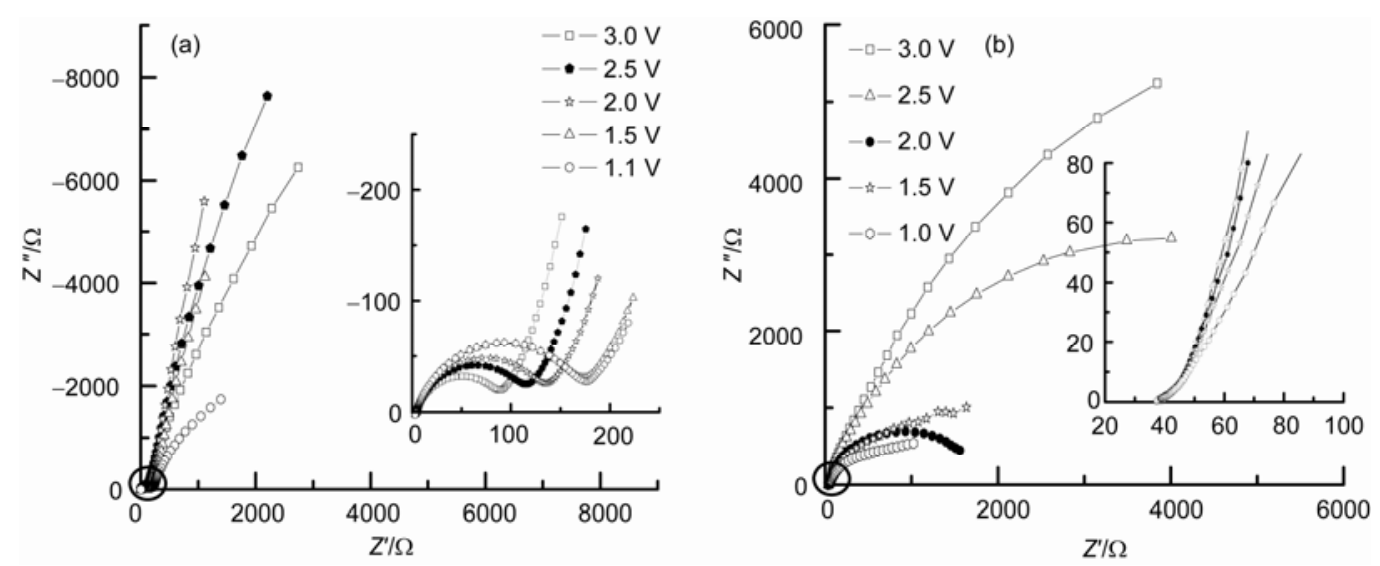

图 3 石墨电极在 $1 \mathrm{~mol} \cdot \mathrm{L}^{-1} \mathrm{LiPF}_{6}$-EC:DEC: DMC 电解液中首次阴极极化过程的 Nyquist 图随电极电位 $(3.0 ~ 1.0 \mathrm{~V})$ 变化

(a) 两电极扣式电池; (b) 三电极玻璃电解池

不断增长. 因此当电极极化电位大于 $1.1 \mathrm{~V}$ 时, EIS的 Nyquist图中HFA应不是由于SEI膜的存在造成的, 而 是由于接触阻抗引起的 ${ }^{[9,14,15]}$. HFA在 3.0 1.5 V之间 的增长, 可能是由于石墨电极在电解液中䛎存时间 的延长或是由于电解液中水等杂质的还原导致接触 阻抗增大造成.

从图 3(b)可以看出, 石墨电极在三电极玻璃电解 池中首次阴极极化时, 在开路电路电位 $3.0 \mathrm{~V}$ 时的 Nyquist图为一段圆弧, EIS图的高频区域不存在HFA. 这与在两电极扣式电池中不同(图 3(a)). 上述结果与 SEM的研究结果一致, 即石墨电极在经历电化学扫 描前其表面不存在初始SEI膜. 随电极极化电位降低 至 $1.0 \mathrm{~V}$, EIS图的高频区域几乎没有发生任何变化, 说明当电极极化电位大于 $1.0 \mathrm{~V}$ 时, 在石墨负极表面 不存在电解液组分的分解、还原形成SEI膜的过程, 这也与电化学循环伏安法的研究结果相一致 ${ }^{[13]}$.

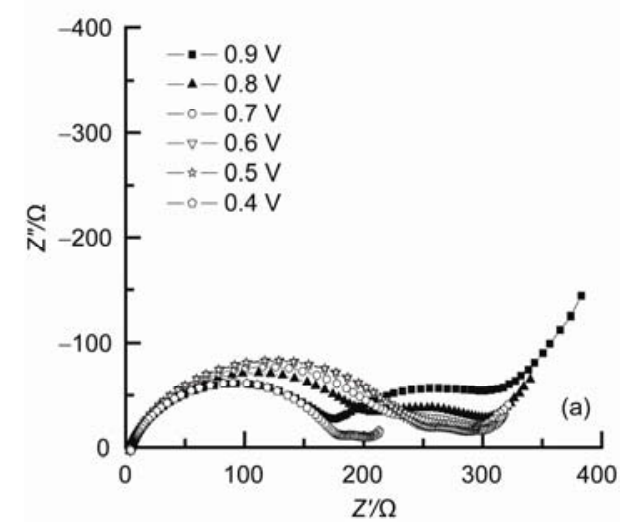

上述结果表明, 在三电极玻璃电解池中不存在 接触阻抗问题, 因而能够有效地消除在两电极或三 电极扣式电池中因接触阻抗引起的 Nyquist 图中高频 区域的 HFA.

如果HFA除了与接触阻抗有关, 还与SEI膜的生 成有关, 那么在SEI膜形成的电位区间, 即 $1.0 \sim 0.5 \mathrm{~V}$ 之 间, 应该能够观察到HFA的增长. Chang等人 ${ }^{[14]}$ 和Martinent等人 ${ }^{[15]}$ 报道, 在SEI膜的主要形成电位区间内, 观察 不到HFA增长. 然而本文的研究结果则不同, 图 4 为石 墨电极在 $1 \mathrm{~mol} \cdot \mathrm{L}^{-1} \mathrm{LiPF} 6-\mathrm{EC}: \mathrm{DEC}$ : DMC电解液中首 次阴极极化过程中 Nyquist图随电极电位 $(0.9 \sim 0.4 \mathrm{~V})$ 的变化. 从图 4(a)可以观察到, 石墨电极在两电极扣 式电池中, 当极化电位低于 $0.9 \mathrm{~V}$ 时, EIS的Nyquist图 主要由三部分组成, 即HFA、中频区域与电荷传递过 程有关的半圆和低频区域反映锂离子在石墨电极中 的固态扩散的斜线. 其中, H F A 在 $0.9 \sim$

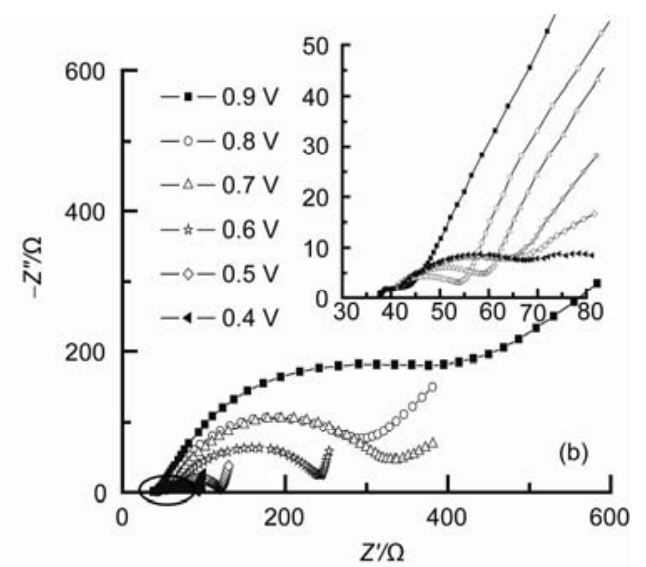

图 4 石墨电极在 $1 \mathrm{~mol} \cdot \mathrm{L}^{-1} \mathrm{LiPF}_{6}-\mathrm{EC}: \mathrm{DEC}: \mathrm{DMC}$ 电解液中首次阴极极化过程的 Nyquist 图随电极电位 $(0.9 \sim 0.4 \mathrm{~V})$ 的变化 (a) 两电极扣式电池; (b) 三电极玻璃电解池 
$0.6 \mathrm{~V}$ 之间随电极极化电位降低不断增长. 由此可以 推知当电极极化电位小于 $0.9 \mathrm{~V}$ 时, HFA 除了与接触 阻抗有关外，可能也与 SEI 膜有关. 在电极极化电位 进一步降低的过程中, 在 0.6 0.5 V 之间, HFA 基本保 持不变; 然而在 0.5 0.4 V 之间, HFA 减小. 因为 SEI 膜的阻抗 随电极极化电位的降低应该是增大或不变 的, 可以推测在 $0.5 \sim 0.4 \mathrm{~V}$ 之间, HFA 的减小是接触阻 抗的变化引起的, 由于接触阻抗随电极极化电位的 变化而改变, 使得我们无法观察到 SEI 膜阻抗随电极 极化电位的具体变化.

从图 4(b)可以看出, 与在两电极扣式电池中不同, 石墨电极在三电极玻璃电解池中首次阴极极化时, 极化电位为 $0.9 \mathrm{~V}$ 时的Nyquist图中最重要的特征是在 高频区域出现了 HFA. 这与人们普遍接受的事实 [16 18], 即SEI膜在 1.0 0.8 V之间开始形成是一致的. 因此, 毫无疑问, 在三电极玻璃电解池中HFA应该是 与SEI膜相关的半圆. 随着电极电位进一步降低, HFA 在 $0.9 \sim 0.6 \mathrm{~V}$ 之间快速增长, 指示SEI膜不断增厚. 在 0.6 0.4 V之间, HFA基本保持不变, 说明此时石墨电 极表面已经形成了完整的SEI膜, 它能够阻止电解液 组分在石墨负极表面上进一步发生还原、分解形成 SEI膜的过程. 上述结果表明, 石墨负极首次阴极极 化过程中SEI膜主要在 1.0 0.6 V区间形成, 这与电化 学循环伏安法观察到对应SEI膜形成的电流峰出现在 $0.5 \mathrm{~V}$ 左右相一致 ${ }^{[13]}$.

石墨电极在 $1 \mathrm{~mol} \cdot \mathrm{L}^{-1} \mathrm{LiPF}_{6}$-EC:DEC:DMC 电 解液中首次阴极极化过程的 Nyquist 图随电极电位 $(0.3 \sim 0.1 \mathrm{~V})$ 的变化如图 5 所示. 从图 5(a)观察到, 在 两电极扣式电池中, HFA 在 $0.3 \sim 0.2 \mathrm{~V}$ 之间增大; 在
$0.2 \sim 0.1 \mathrm{~V}$ 之间减小. 表明接触阻抗受电化学扫描循 环过程的影响较大, 而且随极化电位的变化并不规 律. 从图 5(b)可以看到, 与在两电极扣式电池中不同, 石墨电极在三电极玻璃电解池中的首次阴极极化过 程, HFA 在 $0.3 \sim 0.1 \mathrm{~V}$ 之间基本保持不变, 与在 $0.6 \sim 0.4 \mathrm{~V}$ 之间类似, 说明锂离子在石墨材料嵌脱过 程中 SEI 膜基本保持不变, 从而保证了锂离子在石墨 材料中嵌脱过程的可逆性.

从上述实验结果的分析可以得知, 在两电极扣 式电池中, 石墨电极首次阴极极化过程的 Nyquist 图 中, HFA 除了与 SEI 膜的形成有关外, 还与接触阻抗 有关, 并且在首次阴极极化过程中接触阻抗会随电极 极化电位的变化发生改变. 因此, 在两电极扣式电池 体系中, 测量石墨电极首次阴极极化过程中 EIS 谱特 征的变化, 不能用于研究 SEI 膜的形成过程, 只有在 消除接触阻抗的电池体系, 测量石墨电极首次阴极极 化过程中 EIS 谱特征及其变化, 才有可能揭示 SEI 膜 的形成过程. 在三电极玻璃电解池体系中, Nyquist 图 中的 HFA 归因于 SEI 膜的形成和生长过程. 因此, 运 用 EIS 研究石墨电极在三电极玻璃电解池中的首次阴 极极化过程, 可以获得有关 SEI 膜形成机制的认识.

\section{3 结论}

运用 EIS 并结合 SEM 研究了石墨电极在两电极扣 式电池和三电极玻璃电解池中的首次阴极极化过程. 结果表明, 在两电极扣式电池中, 石墨电极首次阴极 极化过程的 Nyquist 图中, HFA 除了与 SEI 膜的形成有 关外, 还与接触阻抗有关, 并且在首次阴极极化过程中 接触阻抗会随电极极化电位的变化发生改变. 因此, 在 两电极扣式电池体系中, 石墨电极首次阴极极化过程中,
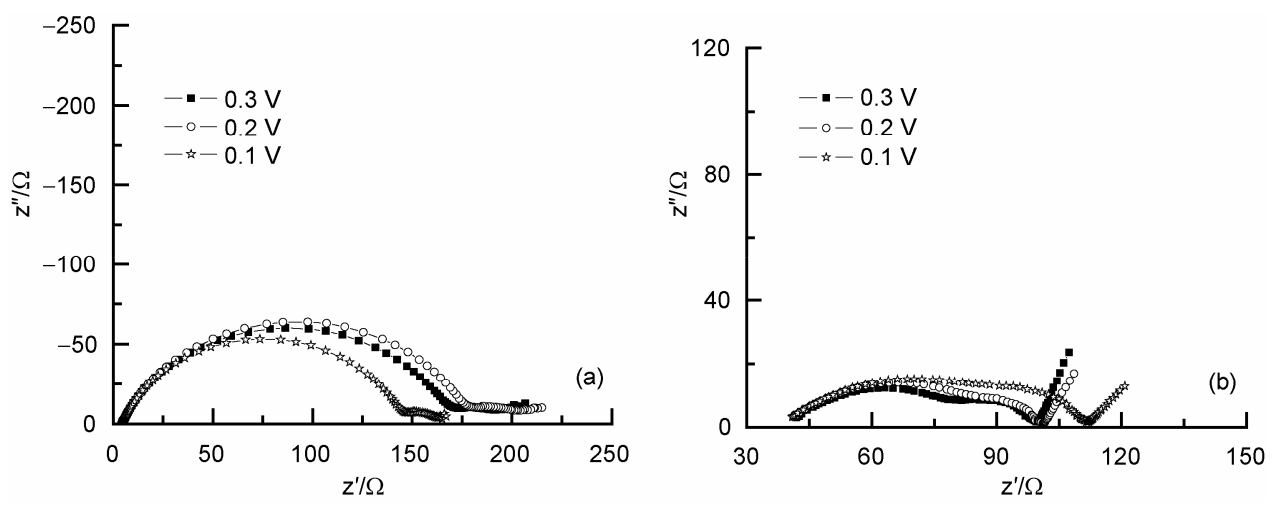

图 5 石墨电极在 $1 \mathrm{~mol} \cdot \mathrm{L}^{-1} \mathrm{LiPF}_{6}$-EC: DEC: DMC 电解液中首次阴极极化过程的 Nyquist 图随电极电位 $(0.3 \sim 0.1 \mathrm{~V})$ 的变化 (a) 两电极扣式电池; (b) 三电极玻璃电解池 
EIS 谱特征的变化不能用于研究 SEI 膜的形成过程; 而在三电极玻璃电解池中能够有效地消除接触阻抗 问题. 因此运用 EIS 研究石墨电极在三电极玻璃电
解池中的首次阴极极化过程, 可用于揭示 SEI 膜的 形成机制. 此外研究结果还指出, 石墨负极首次阴 极极化过程中 SEI 膜主要在 1.0 0.6 V 电位区间形成.

\section{参考文献}

1 Aurbach D. Review of selected electrode-solution interactions which determine the performance of Li and Li ion batteries. J Power Sources, 2000, 89: 206-218 $\underline{\text { [doi] }}$

2 Gnanaraj J S, Thompson R W, Iaconatti S N, et al. Formation and growth of surface films on graphitic anode materials for Li-ion batteries. Electrochem Solid-State Lett, 2005, 8(2): A128-A132[doi]

3 Piao T, Park S M, Doh C H, et al. Intercalation of lithium ions into graphite electrodes studied by AC impedance measurements. J Electrocheml Soc, 1999, 146 (8): 2794-2798[doi]

4 Aurbach D, Gamolsky K, Markovsky B, et al. The study of surface phenomena related to electrochemical lithium intercalation into $\mathrm{Li}_{x} \mathrm{MO}_{y}$ host materials(M=Ni, Mn). J Electrochem Soc, 2000, 147(4): 1322-1331 [doi]

5 Mantia F L, Vetter J, Novák P. Impedance spectroscopy on porous materials: A general model and application to graphite electrodes of lithium-ion batteries. Electrochimica Acta, 2008, 53(12): 4109—4121 [doi]

6 Levi M D, Aurbach D. Simultaneous measurements and modeling of the electrochemical impedance and the cyclic voltammetric characteristics of graphite electrodes doped with lithium. J Phys Chem B, 1997, 101(23): 4630 - 4640[doi]

7 Song J Y, Lee H H, Wang Y Y, et al. Two- and three electrode impedance spectroscopy of lithium-ion batteries. J Power Sources, 2002, 111: 255一267 [doi]

8 庄全超, 许金梅, 樊小勇, 等. $\mathrm{LiCoO}_{2}$ 正极电子和离子传输特性的 EIS 研究. 科学通报, 2007, 52(2): 147-153

9 Holzapfel M, Martinent A, Allion F, et al. First lithiation and charge/discharge cycles of graphite materials, investigated by electrochemical impedance spectroscopy. J Electroanal Chem, 2003, 546: 41-50[doi]

10 Zhang S, Shi P. Electrochemical impedance study of lithium intercalation into MCMB electrode in a gel electrolyte. Electrochimica Acta, 2004, 49: 1475-1482

11 Aurbach D, Markovsky B, Nimberger A, et al. Electrochemical Li-insertion processes into carbons produced by milling graphitic powders: The impact of the carbons' surface chemistry. J Electrochem Soc, 2002, 149(2): A152-A161 [doi]

12 Gnanaraj J S, Levi M D, Levi E, et al. Comparison between the electrochemical behavior of disordered carbons and graphite electrodes in connection with their structure. J Electrochem Soc, 2001, 148(6): A525-A536 [doi]

13 庄全超, 陈作锋, 董全峰, 等. 锂离子电池电解液中甲醇杂质对石墨电极性能影响机制的电化学阻抗谱研究. 高等学校化 学学报, 2005, 26(11): 2073-2076

14 Chang Y C, Sohn H J. Electrochemical impedance analysis for lithium ion intercalation into graphitized carbons. J Electrochem Soc, 2000, 147(1): 50-58[doi]

15 Martinent A, Le Gorrec B, Montella C, et al. Three-electrode button cell for EIS investigation of graphite electrode. J Power Sources, 2001, 97-98: 83-86 [doi]

16 Naji A, Ghanbaja J, Humbert B, et al. Electroreduction of graphite in $\mathrm{LiClO}_{4}$-ethylene carbonate electrolyte. Characterization of the passivating layer by transmission electron microscopy and Fourier-transform infrared spectroscopy. J Power Sources, 1996, 63: 3339 [doi]

17 Wang C, Appleby A J, Little F E. Irreversible capacities of graphite anode for lithium-ion batteries. J Electroanal Chem, 2002, 519: $9-17 \underline{\text { [doi] }}$

18 Chusid O, Ein-Ely E, Aurbach D, et al. Electrochemical and spectroscopic studies of carbon electrodes in lithium battery electrolyte systems. J Power Sources, 1993, 43(1-3): 47-64[doi] 